\title{
Particle Motion in the Stable Region Near an Edge of a Linear Half-Integer Stopband
}

\author{
George Parzen
}

July 20, 1995

BNL-62036

\begin{abstract}
This paper studies the motion of a particle whose tune is inside and near a linear half-integer stopband. Results are found for the tune and beta functions in the stable region close to an edge of the stopband. It is shown that the eigenvalues and the eigenfunctions of the transfer matrix are real inside the stopband. All the results found are also valid for small accelerators where the large accelerator approximation is not used.
\end{abstract}

\section{Introduction}

Inside a linear-half integer stopband the particle motion can be unstable and grow exponentially. A result is found for the growth parameter $g$ which is the imaginary part of the tune. It is shown that the real part of the tune inside the stopband is constant at the value $q / 2$, where $\nu=q / 2$ is the center of the stopband, $q$ being some integer. The proof given does not depend on perturbation theory, and the result follows from the symplectic properties. The eigenvalues and eigenfunctions of the transfer matrix are shown to be real inside the stopband. In the stable region near an edge of the stopband, the tune varies rapidly and the beta function becomes infinite as the unperturbed tune approaches the edge of the stopband. Results are found for the tune and beta function in the stable region near an edge of the stopband. It is found that the beta function becomes infinite inversely as the square root of the distance of the unperturbed tune from the edge of the stopband. 
The basic equations used are also valid for small accelerators, where the large accelerator approximations is not used, and all the results found are valid for small accelerators.

\section{Results when the tune is not in the stopband}

Before treating the interesting case where the tune is inside the stopband, it will be helpful to first treat the case where the tune is not inside the stopband. It will then become clear where the perturbation solution breaks down, when the tune is inside the stopband, and how the perturbation solution can be repaired.

It will be assumed that in the absence of the perturbing fields, the tune of the particle is $\nu_{0}$ and that the motion is stable when $\nu_{0}$ is close to $q / 2$, where $q$ is an integer.

It is assumed that a perturbing field is present which is given on the median plane by

$$
\Delta B_{y}=-G(s) x
$$

$G(s)$ is periodic in $s$ and contains the field harmonics that can excite the stopband around $\nu_{0}=q / 2$.

Introducing $\eta$ defined by

$$
\eta=x / \beta^{1 / 2}
$$

where $\beta$ is the beta function of the unperturbed field, the equation of motion can be written as

$$
\begin{aligned}
& \frac{d^{2} \eta}{d \theta^{2}}+\nu_{0}^{2} \eta=f \\
& f=\nu_{0}^{2} \beta^{3 / 2} \Delta B_{y} / B \rho \\
& f=-\nu_{0}^{2} \beta^{2} G \eta / B \rho \\
& B \rho=p c / e, d \theta=d s / \nu_{0} \beta
\end{aligned}
$$

Eqs. (2-3) are valid for large accelerators, and a small change is required ${ }^{1}$ to make them valid for small accelerators (see section 7). However, the final results found below are valid for small accelerators that require the use of the exact linearized equations. This is shown in section 7 . 
Eq. (2-3) can be written as

$$
\begin{aligned}
\frac{d^{2} \eta}{d \theta^{2}}+\nu_{0}^{2} & =-2 \nu_{0} b(\theta) \eta \\
b(\theta) & =\frac{1}{2} \nu_{0} \beta^{2} G / B \rho
\end{aligned}
$$

Because $b(\theta)$ is periodic a solution for $\eta$ will have the form

$$
\eta=\exp \left(i \nu_{s} \theta\right) h(\theta)
$$

where $h(\theta)$ is periodic. It is assumed that the tune $\nu_{0}$ will change to $\nu_{s}$ because of the perturbing field. Thus $\eta$ can be assumed to have the form

$$
\begin{aligned}
\eta & =A_{s} \exp \left(i \nu_{s} \theta\right)+\sum_{r \neq s} A_{r} \exp \left(i \nu_{r} \theta\right) \\
\nu_{r} & =\nu_{s}+n
\end{aligned}
$$

where $n$ is some integer but $n \neq 0$. For a zero perturbing field the solution for $\eta$ is $\eta=A \exp \left(i \nu_{0} \theta\right)$. Thus for small perturbing fields it can be assumed that

$$
\begin{aligned}
& \nu_{s} \simeq \nu_{0} \\
& A_{r} \ll A_{s} \text { for } r \neq s
\end{aligned}
$$

Putting Eq. (2-6) into Eq. (2-4), one obtains a set of equations for the $A_{r}$

$$
\begin{aligned}
\left(\nu_{r}^{2}-\nu_{0}^{2}\right) A_{r} & =2 \nu_{0} \sum_{\bar{r}} b_{r \bar{r}} A_{\bar{r}} \\
b_{r \bar{r}} & =\frac{1}{2 \pi} \int_{0}^{2 \pi} d \theta b(\theta) \exp \left(-i \nu_{r} \theta+i \nu_{\bar{r}} \theta\right) \\
\nu_{r} & =\nu_{s}+n
\end{aligned}
$$

Eqs. (2-8) are a set of homogenious equations for the $A_{r}$. The condition required for a solution to exist is that the determinant of the coefficients of the $A_{r}$ should vanish. This condition will determine $\nu_{s}$, and then Eq. (2-8) can be solved for the $A_{r}$ in terms of $A_{s}$. It is more convenient to solve Eqs. (2-8) using an iterative perturbation procedure. For the initial guess for $\eta$ in this iterative procedure, one can assume

$$
\eta=A_{s} \exp \left(i \nu_{s} \theta\right)
$$

One can put this result for $\eta$ in the right hand side of Eq. (2-8), and solve for $A_{r}$ which gives

$$
\begin{aligned}
\left(\nu_{r}^{2}-\nu_{0}^{2}\right) A_{r} & =2 \nu_{0} b_{r s} A_{s} \\
\nu_{r} & =\nu_{s}+n
\end{aligned}
$$


For $r=s$, Eq. (2-10) gives

$$
\left(\nu_{s}^{2}-\nu_{0}^{2}\right) A_{s}=2 \nu_{0} b_{s s} A_{s}
$$

which determines $\nu_{s}$. One finds

$$
\nu_{s}-\nu_{0}=b_{s s}
$$

which can be written as

$$
\begin{aligned}
\nu_{s}-\nu_{0} & =b_{0} \\
b_{0} & =\frac{1}{2 \pi} \int_{0}^{2 \pi} d \theta b(\theta) \\
b_{0} & =\frac{1}{4 \pi} \int_{0}^{L} \beta G / B \rho
\end{aligned}
$$

This is the well known result ${ }^{2,3}$ for the first order tune shift due to a gradient $G$. The solution for $\eta$ to first order can be found using Eq. (2-6) and Eq. (2-8)

$$
\begin{aligned}
& \eta=A_{s}\left\{\exp \left(i \nu_{s} \theta\right)+\sum_{r \neq s} \frac{2 \nu_{0}}{\nu_{r}^{2}-\nu_{0}^{2}} b_{r s} \exp \left(i \nu_{r} \theta\right)\right\} \\
& \nu_{r}=\nu_{s}+n, \quad n \neq 0
\end{aligned}
$$

which can be also written as

$$
\begin{aligned}
\eta & =A_{s} \exp \left(i \nu_{s} \theta\right)\left\{1+\sum_{n \neq 0} \frac{2 \nu_{0} b_{n}}{n\left(n+2 \nu_{0}\right)} \exp (i n \theta)\right\} \\
b_{n} & =\frac{1}{4 \pi} \int_{0}^{L} d s \beta G \exp (-i n \theta) / B \rho
\end{aligned}
$$

One can see from Eq. (2-13b) that the above solution will not be valid when $\nu_{0}$ is close to a half-integer for the denominator $\left(n+2 \nu_{0}\right)$ can become very small.

\section{Results when the tune is inside the stopband}

It is assumed that $\nu_{0}$ is near $\nu_{0}=q / 2, q$ being an integer. The stopband is defined as the range of $\nu_{0}$ for which the tune, $\nu_{s}$, in the presence of the perturbation given by Eq. (2-2) has a non-zero imaginary part. One can write $\nu_{s}$ as

$$
\nu_{s}=\nu_{s}-i g
$$

It will be shown that inside the stopband, where $g \neq 0$, then

$$
\nu_{s R}=q / 2
$$


This may be shown as follows. Let $\mu$ be the phase shift for a period, and $\mu=2 \pi \nu_{s}$ where the period has been assumed to be one turn or $2 \pi$ in $\theta$. Let $T$ be the transfer matrix for one period. Then one has

$$
\cos \mu=\frac{1}{2}\left(T_{11}+T_{22}\right)
$$

and one sees that $\cos \mu$ is real even inside the stopband since the $T_{i j}$ are real. One also has

$$
\cos \mu=\cos 2 \pi\left(\nu_{s R}-i g\right)=\cos \left(2 \pi \nu_{s R}\right) \cosh (2 \pi g)+i \sin \left(2 \pi \nu_{s R}\right) \sinh 2 \pi g
$$

In order for $\cos \mu$ to be real, one has to have either $g=0$ or, if $g \neq 0,2 \pi \nu_{s R}=n \pi$, where $n$ is some integer. Thus, inside the stopband where $g \neq 0$,

$$
\nu_{s R}=n / 2
$$

In order to have continuity when the perturbation goes to zero, one has $n=q$ and

$$
\nu_{s R}=q / 2
$$

For the range of $\nu_{0}$ which is inside the stopband, $\nu_{s R}$ remains constant at $\nu_{s R}=q / 2$ while $g$ goes, as will be seen below, from $g=0$ at the edge of the stopband to a maximum value in the middle of the stopband, where $\nu_{0}=q / 2$. One may note that this result does not depend on the use of perturbation theory and is valid even for large perturbations. A proof of the result, based on perturbation theory, is given below.

Now let us return to the problem of computing the growth factor, $g$, using perturbation theory. The iterative perturbation approach used in section 2 appears to breakdown when $\nu_{0}$ is close to $q / 2$. One sees from Eq. (2-10) that when $\nu_{0} \simeq q / 2$, then one of the $A_{r}$ becomes comparable to $A_{s}$, and this is the $A_{r}$ for which $\nu_{r}=\nu_{s}-q, \nu_{r} \simeq-q / 2$. Thus in the above iterative procedure for finding $\eta$, one will assume for the initial guess for $\eta$, instead of Eq. (2-9),

$$
\begin{aligned}
\eta & =A_{s} \exp \left(i \nu_{s} \theta\right)+A_{\bar{s}} \exp \left(i \nu_{\bar{s}} \theta\right) \\
\nu_{\bar{s}} & =\nu_{s}-q
\end{aligned}
$$

Then Eq. (2-10) is replaced by

$$
\begin{aligned}
\left(\nu_{r}^{2}-\nu_{o}^{2}\right) A_{r} & =2 \nu_{0} b_{r s} A_{s}+2 \nu_{0} b_{r \bar{s}} A_{\bar{s}} \\
\nu_{r} & =\nu_{s}+n \quad \text { or } \quad \nu_{r}=\nu_{\bar{s}}+n=\nu_{s}-q+n
\end{aligned}
$$


For $r=s$ and $r=\bar{s}$ one obtains 2 equations for $A_{s}$ and $A_{\bar{s}}$

$$
\begin{aligned}
\left(\nu_{s}^{2}-\nu_{0}^{2}\right) A_{s} & =2 \nu_{0} b_{s \bar{s}} A_{\bar{s}} \\
\left(\nu_{\bar{s}}^{2}-\nu_{0}^{2}\right) A_{\bar{s}} & =2 \nu_{0} b_{\bar{s} s} A_{s} \\
\nu_{\bar{s}} & =\nu_{s}-q
\end{aligned}
$$

In Eq. (3-9), it has been assumed, for simplicities sake, that $b_{s s}=0$. This can be accomplished by redefining $\nu_{0}$ to be $\nu_{0}+b_{0}$.

In order for Eqs. (3-9) to have a solution, one must have

$$
\begin{aligned}
\left(\nu_{s}^{2}-\nu_{0}^{2}\right)\left(\nu_{\bar{s}}^{2}-\nu_{0}^{2}\right) & =4 \nu_{0}^{2}\left|b_{s \bar{s}}\right|^{2} \\
\nu_{\bar{s}} & =\nu_{s}-q
\end{aligned}
$$

Eq. (3-10) determines $\nu_{s}$. If one writes $\nu_{s}=\nu_{s R}-i g$, one finds

$$
\left[\left(\nu_{s R}-i g\right)^{2}-\nu_{0}^{2}\right]\left[\left(\nu_{s R}-q-i g\right)^{2}\right]=4 \nu_{0}^{2}|\Delta \nu|^{2}
$$

where $\Delta \nu=b_{s \bar{s}}$

$$
\Delta \nu=\frac{1}{4 \pi} \int_{0}^{L} d s \beta \exp (-i q \theta) G / B \rho
$$

$|\Delta \nu|$ will turn out to be the half width of the stopband. Assuming that $\nu_{0}$ and $\nu_{s R}$ are close to $q / 2$, then Eq. (3-11a) can be rewritten as

$$
\left(\nu_{s R}-i g-\nu_{0}\right)\left(q-\nu_{s R}+i g-\nu_{0}\right)=|\Delta \nu|^{2}
$$

The imaginary part of the left hand side gives the equation,

$$
g\left(2 \nu_{s}-q\right)=0
$$

Inside the stopband where $g \neq 0$, one gets

$$
\nu_{s R}=q / 2
$$

Using Eq. (3-14) for $\nu_{s R}$, the real part of Eq. (3-12) gives

$$
\begin{aligned}
\left(q / 2-\nu_{0}\right)^{2}+g^{2} & =|\Delta \nu|^{2} \\
g & = \pm\left\{|\Delta \nu|^{2}-\left(q / 2-\nu_{0}\right)^{2}\right\}^{1 / 2}
\end{aligned}
$$

Eq. (3-15) shows that the growth parameter $g$ has a maximum at $\nu_{0}=q / 2$ where $g=|\Delta \nu|$, and decreases to zero at $\nu_{0}=q / 2 \pm|\Delta \nu|$. The stopband width is then $2|\Delta \nu|$ and extends from $q / 2-|\Delta \nu|$ to $q / 2+|\Delta \nu|$. Thus $|\Delta \nu|$ is the half-width of the stopband. 
Now let us find the solutions for $\eta$ that will give the particle motion inside the stopband. To lowest order, $\eta$ is given by Eq. (3-7) and inside the stopband

$$
\begin{aligned}
\eta & =A_{s} \exp \left(i \nu_{s} \theta\right)+A_{\bar{s}} \exp \left(i \nu_{\bar{s}} \theta\right) \\
\nu_{s} & =q / 2-i g \\
\nu_{\bar{s}} & =-q / 2-i g
\end{aligned}
$$

$A_{s}$ and $A_{\bar{s}}$ are related by Eqs. (3-9) which gives

$$
\begin{aligned}
A_{\bar{s}} & =\frac{2 \nu_{0} b_{\bar{s} s}}{(q / 2+i g)^{2}-\nu_{0}^{2}} A_{s} \\
A_{\bar{s}} & =\frac{b_{\bar{s} s}}{\left(q / 2-\nu_{0}\right)+i g} A_{s}
\end{aligned}
$$

where it has been assumed that $\nu_{0}$ is close to $q / 2$. Eq. (3-17) can be written as

$$
\begin{aligned}
A_{\bar{s}} & =A_{s} \exp \left[-i\left(\delta_{1}+\delta_{2}\right)\right] \\
\delta_{1} & =\text { phase } \Delta \nu \\
\delta_{2} & =\text { phase }\left[\left(q / 2-\nu_{0}\right)+i g\right]
\end{aligned}
$$

where $b_{\bar{s} s}=|\Delta \nu| \exp \left(-i \delta_{1}\right)$, and $|\Delta \nu|=g^{2}+\left(q / 2-\nu_{0}\right)^{2}$ were used.

Putting these results for $A_{\bar{s}}$ into Eq. (3-16) one gets for $\eta$ and $x$

$$
\begin{aligned}
\eta & =A_{s} \exp (g \theta) \cos \left(q \theta / 2-\left(\delta_{1}+\delta_{2}\right) / 2\right) \\
\delta_{1} & =\text { phase } \Delta \nu \\
\delta_{2} & =\text { phase }\left[\left(q / 2-\nu_{0}\right)+i g\right] \\
x & =\beta^{1 / 2} \eta
\end{aligned}
$$

where the multiplying constant $\exp \left[-i\left(\delta+\delta_{2}\right) / 2\right]$ was dropped. Eq. (3-19) give to lowest order the two solutions inside the stopband corresponding to whether $g$ is positive or negative. The first order correction to $\eta$ could also be found using Eq. (3-8) and the result is

$$
\begin{aligned}
\eta & =A_{s} \exp (g \theta)\left[\cos \left(q \theta / 2-\left(\delta_{1}+\delta_{2}\right) / 2\right)\right. \\
& \left.+\sum_{n \neq 0,-q} \frac{2 \nu_{0}}{n\left(n+2 \nu_{0}\right)} b_{n} \cos \left((q / 2+n) \theta-\left(\delta_{1}+\delta_{2}\right) / 2\right)\right] \\
b_{n} & =\frac{1}{4 \pi} \int_{0}^{L} d s \beta G \exp (-i n \theta) / B \rho
\end{aligned}
$$


The results found above for the stopband width, the growth rate and particle motion in the stopbands will apply equally well to small accelerators where the exact linear equations have to be used, without the approximations used for large accelerators, as shown in section 7.

\section{The realness of the eigenvalues and eigenvectors inside the stopband}

It will be shown in this section that the eigenvalues and the eigenvectors of the one period transfer matrix are real when $\nu_{0}$ is inside the stopband. If $\hat{\mathrm{T}}(s)=\mathrm{T}(s+L, s)$ is the one period transfer matrix, then the eigenvalues and eigenvectors satisfy the equation

$$
\begin{aligned}
\hat{\mathrm{T}}(s) x(s) & =\lambda x(s) \\
x & =\left[\begin{array}{c}
x \\
p_{x}
\end{array}\right]
\end{aligned}
$$

The symbol $x$ is used to indicate both the column vector $x$ and the first element of this vector. The meaning of $x$ should be clear from the context. The eigenvalue $\lambda$ is related to $\nu$-value by

$$
\begin{aligned}
& \lambda=\exp (i \mu) \\
& \mu=2 \pi \nu
\end{aligned}
$$

where, for simplicity, the period is assumed to be one turn or $2 \pi$ in $\theta$.

In section 3 it was shown that when $\nu_{0}$ is inside the stopband, $\nu$ can be written as $\nu=q / 2-i g$ one finds for $x$, when $\nu_{0}$ is inside the stopband,

$$
\begin{aligned}
& \lambda=\exp (i q \pi+2 \pi g) \\
& \lambda=(-1)^{q} \exp (2 \pi g)
\end{aligned}
$$

and the eigenvalue, $\lambda$, is real when $\nu_{0}$ is inside the stopband.

Now let us consider the eigenvectors of $\hat{\mathrm{T}}$. If $\lambda, x$ are an eigenvalue and eigenvector of $\hat{\mathrm{T}}$, it is known that $1 \lambda$ is an eigenvalue of $\hat{\mathrm{T}}$, as $\hat{\mathrm{T}}$ is symplectic. Since $\hat{\mathrm{T}}$ is real, $\lambda^{*}, x^{*}$ are also an eigenvalue and eigenvector respectively of $\hat{\mathrm{T}}$. It appears that one has 3 eigenvalues, $\lambda, 1 / \lambda$ and $\lambda^{*}$ instead of just two. Two of the 3 apparent eigenvalues must be equal.

When $\nu_{0}$ is not in the stopband then $\nu_{s}$ is real, $1 / \lambda=\exp (-i \mu)=\lambda^{*}$, and the second solution is $\lambda^{*}, x^{*}$. 
When $\nu_{0}$ is in the stopband, then $\nu$ has an imaginary part, $\nu=q / 2-i g$. Then $1 / \lambda \neq \lambda^{*}$. To avoid having 3 eigenvalues, one needs to have $\lambda^{*}=\lambda$ and $x^{*}=x$, or $\lambda$ and $x$ are real when $\nu_{0}$ is in the stopband.

One should note that the statement $x$ is real means here that $x$ can be made real by multiplying by a constant. This is because an eigenvector can be multiplied by a constant and still be an eigenvector of $\hat{\mathrm{T}}$. Thus the eigenvector may not appear real because of the presence of a constant complex multiplier, but can be made real by multiplying it with a constant.

In section 3 , where $\eta$ was found by an iterative perturbation procedure for $\nu_{0}$ in the stopband, the solution found, Eq. (3-17), is real as required by the above theorm, whereas when $\nu_{0}$ is not inside the stopband, the solution for $\eta$ found Eq. (2-13) is complex. Since the solution for $\eta$ was specified to have the form given by Eq. (2-6), then $x=\eta / \beta^{1 / 2}$ is the first element of the eigenvector, $x$, of $\hat{\mathrm{T}}$.

\section{Tune near the edge of a stopband}

In this section, a result will be found for the tune in the stable region outside the stopband but close to one of the edges of the stopband. It will be shown that close to the edge of a stopband,

$$
|\nu-q / 2|=\left\{2|\Delta \nu|\left|\nu_{0}-\nu_{e}\right|\right\}^{1 / 2}
$$

$\nu$ is the tune in the presence of the gradient perturbation, $\nu_{e}$ is the edge of the stopband, $\nu_{e}=q / 2 \pm|\Delta \nu| .|\Delta \nu|$ is the half-width of the stopband. Eq. (5-1) shows that when $\nu_{0}$ is close to an edge of the stopband, $\nu_{e}, \nu$ varies rapidly with $\nu_{0}$, and the slope of the $\nu$ vs. $\nu_{0}$ curve is vertical at $\nu_{0}=\nu_{e}$.

To find $\nu$ in the stable region outside the stopband, where $\nu_{0}-q / 2|>| \Delta \nu \mid$, one goes back to the derivation given in section 3 for $\nu$ inside the stopband, starting with Eq. (3-12). Eq. (3-15) shows that for $\left|\nu_{0}-q / 2\right|>|\Delta \nu|$ the only acceptable solution is $g=0$, and Eq. (3-12) can be written as

$$
\left(\nu-\nu_{0}\right)\left(|\nu-q|-\nu_{0}\right)=|\Delta \nu|^{2}
$$

where we have put $\nu_{s}=\nu$. 
Assuming that $\nu_{0}$ is just below the stopband edge $\nu_{e}=q / 2-|\Delta \nu|$, put $\nu_{0}=\nu_{e}-\epsilon$ and $\nu=q / 2-\delta$ into Eq. (5-2), where $\epsilon$ and $\delta$ both approach zero as $\nu_{0}$ approaches the stopband edge. We find

$$
\delta=\{\epsilon(\epsilon+2|\Delta \nu|)\}^{1 / 2}
$$

The top edge of the stopband can be treated in the same way and both results can be combined into the one result

$$
\begin{aligned}
|\nu-q / 2| & =\left\{\left|\nu_{0}-\nu_{e}\right|\left(\left|\nu_{0}-\nu_{e}\right|+2|\Delta \nu|\right)\right\}^{1 / 2} \\
\nu_{e} & =q / 2 \pm|\Delta \nu|
\end{aligned}
$$

Very close to the stopband edge, $\left|\nu_{0}-\nu_{e}\right| \ll|\Delta \nu|$, one finds

$$
|\nu-q / 2|=\left\{2|\Delta \nu|\left|\nu_{0}-\nu_{e}\right|\right\}^{1 / 2}
$$

Thus, as $\nu_{0}$ approaches a stopband edge, $\nu$ approaches $q / 2$, and $d \nu / d \nu_{0}$ become infinite like $1 /\left|\nu_{0}-\nu_{e}\right|^{1 / 2}$.

\section{The beta function near the edge of the stopband}

In this section, a result will be found for the beta function in the stable region outside the stopband, but close to one of the edges of the stopband. It will be shown that close to edge of a stopband

$$
\left[\left(\beta-\beta_{0}\right) / \beta_{0}\right]_{\max }=\left[2|\Delta \nu| /\left|\nu_{0}-\nu_{e}\right|\right]^{1 / 2}
$$

$\nu_{e}$ is the edge of the stopband, $\nu_{e}=q / 2 \pm|\Delta \nu| .|\Delta \nu|$ is the half-width of the stopband. $\nu_{0}$, $\beta_{0}$ are the unperturbed tune and beta function. Eq. (6-1) shows that when $\nu_{0}$ approaches the edge of the stopband, $\left(\beta-\beta_{0}\right) / \beta_{0}$ becomes infinite like $1 /\left|\nu_{0}-\nu_{e}\right|^{1 / 2}$.

The beta function $\beta$ can be found from the solution for the $\eta$ function which has the form given by Eq. (2-5). It will be shown below that $\beta$ is given by

$$
\begin{gathered}
\frac{\beta}{\beta_{0}}=\frac{\nu_{0}}{\nu}\left|\eta^{2}\right|\left\langle\frac{1}{|\eta|^{2}}\right\rangle \\
\left\langle\frac{1}{|\eta|^{2}}\right\rangle=\frac{1}{2 \pi} \int_{0}^{2 \pi} d \theta \frac{1}{|\eta|^{2}}
\end{gathered}
$$

$\nu, \beta$ and $\nu_{0}, \beta_{0}$ are the perturbed and unperturbed values of the tune and the beta function. Thus $\beta$ can be determined from the $\eta$ function, if the perturbed tune is known, and in this case the tune was found in section 5 . 
To derive Eq. (6-2) one notes that

$$
x=\beta_{0}^{1 / 2} \eta,
$$

and thus

$$
\beta=D \beta_{0}|\eta|^{2}
$$

where $D$ is some constant. The constant $D$ can be determined using the relationship

$$
\frac{\nu}{\nu_{0}}=\frac{1}{2 \pi} \int_{0}^{2 \pi} d \theta \frac{\beta_{0}}{\beta}
$$

which when written in this form, will hold also for small accelerators ${ }^{1}$ when the perturbation does not change the closed orbit (see section 7). This gives

$$
\begin{aligned}
D & =\frac{\nu_{0}}{\nu}\left\langle 1 /|\eta|^{2}\right\rangle \\
\left(\beta / \beta_{0}\right) & =\left(\nu_{0} / \nu\right)|\eta|^{2}\left\langle 1 /|\eta|^{2}\right\rangle
\end{aligned}
$$

One now proceeds to find the $\eta$ function in the stable region near an edge of the stopband. To lowest order, $\eta$ is given by Eq. (3-7) as

$$
\begin{aligned}
\eta & =A_{s} \exp \left(i \nu_{s} \theta\right)+A_{\bar{s}} \exp \left(i \nu_{\bar{s}} \theta\right) \\
\nu_{\bar{s}} & =\nu_{s}-q
\end{aligned}
$$

$A_{\bar{s}}$ is given by Eq. (3-9) which can be written as

$$
\begin{aligned}
A_{\bar{s}} & =\frac{|\Delta \nu| \exp \left(-i \delta_{i}\right)}{\left|\nu_{s}-q\right|-\nu_{0}} A_{s} \\
\delta_{1} & =\operatorname{ph}(\Delta \nu)
\end{aligned}
$$

Assuming $\nu_{0}$ is below the edge of the stopband one puts $\nu_{s}=q / 2-\delta$ and $\nu_{0}=\nu_{e}-\epsilon$, $\nu_{e}=q / 2-|\Delta \nu|$. Then

$$
A_{\bar{s}}=\frac{|\Delta \nu| \exp \left(-i \delta_{1}\right)}{\delta+\epsilon+|\Delta \nu|} A_{s}
$$

The $\eta$ functions is then given to lowest order by

$$
\begin{aligned}
& \eta=A_{s} \exp \left(i \nu_{s} \theta\right)\left[1+\frac{|\Delta \nu|}{|\Delta \nu|+\epsilon+\delta} \exp \left[i\left(-q \theta-\delta_{1}\right)\right]\right] \\
& \epsilon=\left|\nu_{0}-\nu_{e}\right|, \quad \nu_{e}=q / 2 \pm|\Delta \nu| \\
& \delta=|\nu-q / 2|=\left\{\left|\nu_{0}-\nu_{e}\right|\left(\left|\nu_{0}-\nu_{e}\right|+2|\Delta \nu|\right)\right\}^{1 / 2}
\end{aligned}
$$


Eq. (6-9) has been written so that it holds both above and below the stopband, and Eq. (5-4) was used to replace $\delta$.

One then finds

$$
\begin{gathered}
|\eta|^{2}=\left|A_{s}\right|^{2}\left(1+2 C \cos \left(q \theta+\delta_{1}\right)+C^{2}\right) \\
C=\frac{|\Delta \nu|}{|\Delta \nu|+\left|\nu_{0}-\nu_{e}\right|+|\nu-q / 2|} \\
\left\langle\frac{1}{|\eta|^{2}}\right\rangle=\frac{1}{\left|A_{s}\right|^{2}} \frac{1}{2 \pi} \int_{0}^{2 \pi} d \theta \frac{1}{1+2 C \cos \left(q \theta+\delta_{1}\right)+C^{2}} \\
=\frac{1}{\left|A_{s}\right|^{2}} \frac{1}{1-C^{2}}
\end{gathered}
$$

One can now use Eq. (6-2) to find $\beta$

$$
\frac{\beta}{\beta_{0}}=\frac{\nu_{0}}{\nu}\left(1+C^{2}+2 C \cos \left(q \theta+\delta_{1}\right)\right) \frac{1}{1-C^{2}}
$$

One may note that when $\nu_{0}$ is at the edge of a stopband, $\nu_{e}-\nu_{0}=0, \nu-q / 2=0$ and $C=1$. Since one is interested here in the rather large effects due to the $1 /\left(1-C^{2}\right)$ factor near the edge of the stopband, one can put $\nu_{0} / \nu=1$ with only a small error. One finds for $\beta$

$$
\begin{aligned}
\left(\beta-\beta_{0}\right) / \beta_{0} & =\frac{2 C\left(C+\cos \left(q \theta+\delta_{1}\right)\right)}{1-C^{2}} \\
\left(\left(\beta-\beta_{0}\right) / \beta_{0}\right)_{\max } & =\frac{2 C}{1-C} \\
\left(\left(\beta-\beta_{0}\right) / \beta_{0}\right)_{\max } & =\frac{2|\Delta \nu|}{\left|\nu_{0}-\nu_{e}\right|+|\nu-q / 2|}
\end{aligned}
$$

using the result $|\nu-q / 2|=\left\{\left(\nu_{0}-\nu_{e}\right)\left(\left|\nu_{0}-\nu_{e}\right|+2|\Delta \nu|\right)\right\}^{1 / 2}$ one gets

$$
\left(\left(\beta-\beta_{0}\right) / \beta_{0}\right)_{\max }=\frac{2|\Delta \nu|}{\left|\nu_{0}-\nu_{e}\right|+\left\{\left|\nu_{0}-\nu_{e}\right|\left(\left|\nu_{0}-\nu_{e}\right|+2|\Delta \nu|\right)\right\}^{1 / 2}}
$$

Very close to the stopband, $\left|\nu_{0}-\nu_{e} \ll\right| \Delta \nu \mid$, one finds

$$
\left(\left(\beta-\beta_{0}\right) / \beta_{0}\right)_{\max }=\left\{\frac{2|\Delta \nu|}{\left|\nu_{0}-\nu_{e}\right|}\right\}^{1 / 2}
$$

Eq. (6-15) shows that as $\nu_{0}$ approaches the edge of a stopband $\left(\left(\beta-\beta_{0}\right) / \beta_{0}\right) \max$ becomes infinite like $\left\{\left|\nu_{0}-\nu_{e}\right|\right\}^{-1 / 2}$.

Table 1 below shows how the distortion in the beta function depends on how far $\nu_{0}$ is from the edge of stopband. The table shows that to keep $\left(\left(\beta-\beta_{0}\right) / \beta_{0}\right)_{\max }$ less than $10 \%$, then $\nu_{0}$ has to be about $10 \Delta \nu$ away from the stopband edge. 
Table 1: $\left(\left(\beta-\beta_{0}\right) / \beta_{0}\right)_{\max }$ versus $\left|\nu_{0}-\nu_{e}\right| /|\Delta \nu|$ as computed from Eq. (6-14).

\begin{tabular}{|cccccccc|}
\hline$\left|\nu_{0}-\nu_{e}\right| /|\Delta \nu|$ & .5 & 1 & 2 & 4 & 6 & 8 & 10 \\
$\left(\left(\beta-\beta_{0}\right) / \beta_{0}\right)_{\max }$ & 1.24 & .73 & .41 & .22 & .15 & .12 & .095 \\
\hline
\end{tabular}

\section{Comments on the small accelerator results}

All the final results found in this paper will also hold for small accelerators where the exact equations of motions have to be used. The exact linear equations have the form ${ }^{1}$

$$
\begin{aligned}
\frac{d x}{d s} & =A_{11} x+A_{12} q_{x} \\
\frac{d q_{x}}{d s} & =A_{21} x+A_{22} q_{x} \\
q_{x} & =p_{x} / p
\end{aligned}
$$

In the large accelerator approximation, it is assumed that $A_{11}=A_{22}=0, A_{12}=1$, and $q_{x} \simeq d x / d s$. The coefficients $A_{i j}$ are given in reference 1 . In particular, $A_{12}$ is given by

$$
A_{12}=\frac{1+x / \rho}{\left(1-q_{x}^{2}\right)^{3 / 2}}
$$

where the right hand side is evaluated on the closed orbit.

Although for small accelerators, the differential equations for $x$ and $\beta$ are different from those usually used for large accelerators, it has been found ${ }^{1}$ that the linearized differential equation for $\eta=x / \beta^{1 / 2}$ is not much different. One finds

$$
\begin{gathered}
\frac{d^{2}}{d \theta^{2}} \eta+\nu_{0}^{2} \eta=f \\
f=\frac{\nu_{0}^{2} \beta_{0}^{2}}{A_{12}} \frac{\Delta B_{y}}{B \rho} \\
B \rho=p c / e
\end{gathered}
$$

with the difference that $\theta$ is now defined by ${ }^{1}$

$$
d \theta=A_{12} \frac{d s}{\nu_{0} \beta_{0}}
$$

where $\nu_{0}, \beta_{0}$ are the unperturbed tune and beta function. The $A_{12}$ is evaluated on the unperturbed closed orbit. 
The relationshp between $\nu$ and $\beta$ is somewhat different for small accelerators, and is given by ${ }^{1}$

$$
\nu=\frac{1}{2 \pi} \int_{0}^{C} \frac{A_{12}}{\beta} d s
$$

where $C$ is the circumference of the accelerator. Using Eq. (7-4) this can be written as

$$
\frac{\nu}{\nu_{0}}=\frac{1}{2 \pi} \int_{0}^{2 \pi} \frac{A_{12}}{\bar{A}_{12}} \frac{\beta_{0}}{\beta} d \theta
$$

where $\bar{A}_{12}$ is the $A_{12}$ coefficient evaluated on the unperturbed closed orbit. If the perturbation being considered does not change the closed orbit, then $A_{12}=\bar{A}_{12}$ and one has

$$
\frac{\nu}{\nu_{0}}=\frac{1}{2 \pi} \int_{0}^{2 \pi} \frac{\beta_{0}}{\beta} d \theta
$$

Eq. (7-7) now holds for both large and small accelerators, provided the perturbation does not change the closed orbit.

The results found in this paper are based on Eq. (7-3) for $\eta$ and Eq. (7-7) that relates $\nu$ and $\beta$. Although, the perturbation term in Eq. (7-3) now has the extra factor $1 / A_{12}$ for small accelerators, this factor of $1 / A_{12}$ disappears in the final result when one goes from the variable $\theta$ to the variable $s$ according to Eq. (7-4). Thus the result for the stopband width, $\Delta \nu$, as given by Eq. (3-11b) is valid for both large and small accelerators. Keeping the above equations in mind, one can go through the derivations on the previous pages, and show that the final results are valid for both large and small accelerators.

\section{Comments on the results}

Others have worked on this subject and there is an overlap between the contents of this paper and their work. These previous papers (2 to 6 ) give results for the stopband width and for the growth rate inside the stopband.

The results in this paper include the following. The result given for the tune $\nu$ near the edge of the stopband, $\nu_{e},|\nu-q / 2|=\left[2|\Delta \nu|\left|\nu_{e}-\nu_{0}\right|\right]^{1 / 2}$. The result given for the beta function near the edge of the stopband, $\left[\left(\beta-\beta_{0}\right) / \beta_{0}\right]_{\max }=\left[2|\Delta \nu| /\left|\nu_{e}-\nu_{0}\right|\right]^{1 / 2}$. The proof given showing that the real part of $\nu$ is constant over the stopband at $q / 2$ does not depend on perturbation theory, and the result follows from the symplectic properties. The 
result that all the results found in this paper will also hold for a small accelerator where the large accelerator approximation is not used. The result given for the solutions of the equations of motion when $\nu_{0}$ is inside the stopband, and the proof that the eigenfunctions and eigenvalues of the transfer matrix are real inside the stopband.

\section{References}

1. G. Parzen, Linear orbit parameters for the exact equations of motion, BNL Report, BNL-60090 (1994).

2. P.A. Sturrock, Static and dynamic electron optics, Cambridge Univ. Press, London (1955).

3. E.D. Courant and H.S. Snyder, Theory of the alternating gradient synchrotron, Annals. of Physics, Vol. 3, p. 1 (1958).

4. H. Bruck, Circular particle accelerators, (1965), English translation Los Alamos Report, LA-TR-72-10 Rev.

5. A.A. Kolomensky and A.N. Lebedev, Theory of cyclic accelerators, North Holland Publishing Co., Amsterdam (1966).

6. H. Wiedemann, Particle accelerator physics, Springer-Verlag, Berlin (1993). 